\title{
PSMA Theranostics: Is the Time Ripe to Pave the Way to Further Tumor Entities?
}

\author{
Winfried Brenner ${ }^{1,2}$, Joachim Strobel ${ }^{3}$, and Vikas Prasad ${ }^{2,3}$ \\ ${ }^{1}$ Department of Nuclear Medicine, Charité-Universitätsmedizin Berlin, Berlin, Germany; ${ }^{2}$ German Cancer Consortium (DKTK), Campus \\ Berlin, Berlin, Germany; and ${ }^{3}$ Department of Nuclear Medicine Universität Ulm, Ulm, Germany
}

\section{$\mathbf{S}$} individualized tumor characterization. State-of-the-art personalized treatment options are still primarily directed by site of tumor origin and tumor entity rather than by the tumor molecular signature (e.g., the novel treatment approach with neurotrophic tyrosine receptor kinase [NTRK] inhibitors in the case of NTRK gene fusionpositive tumors) irrespective of their origin. Molecular imaging using metabolic tracers and radiolabeled peptides and antibodies offer a unique possibility for noninvasive in vivo tumor characterization, also based on molecular paradigms rather than tumor type. One such radiopharmaceutical, the prostate-specific membrane antigen (PSMA), is currently set to become a blockbuster for both PET diagnostics and radionuclide therapy in prostate cancer. Germany, specifically the University of Heidelberg team, has pioneered the rejuvenation of PSMA and its use in nuclear medicine. The evidence level from initial retrospective German studies was substantiated by subsequent prospective clinical trials in Australia.

The proPSMA diagnostic trial reported that "PSMA PET/CT is a suitable replacement for conventional imaging, providing superior accuracy to the combined findings of CT and bone scanning" in treatment-naïve patients with high-risk prostate cancer with respect to detection of both pelvic nodal and distant metastases $(1,2)$. Similarly, PSMA PET/CT proved also superior in detecting local recurrences, lymph node, or distant metastases in patients with early biochemical relapse and rising prostate-specific antigen levels as low as $0.2 \mathrm{ng} / \mathrm{mL}$ (3). These findings support recent U.S. Food and Drug Administration (FDA) approval of 2 new drug applications for PSMA PET imaging at the Universities of California San Francisco and Los Angeles for both primary staging in high-risk prostate cancer patients and patients with biochemical recurrent disease. The findings also reinforce the recommendation for PSMA PET imaging in the setting of biochemical recurrence in the recently updated national German S3 guideline on prostate cancer (4) and reimbursement of this procedure within the framework of Ambulante Spezialärtzliche Versorgung in Germany. Most recently, the PSMA tracer ${ }^{18} \mathrm{~F}$-piflufolastat from Lantheus was approved by the FDA (5).

Additionally, major progress in the realm of PSMA treatment in patients with prostate cancer has been made. The results of the

Received Jun. 13, 2021; revision accepted Jun. 29, 2021.

For correspondence or reprints, contact Winfried Brenner (winfried. brenner@charite.de).

Published online July 16, 2021

COPYRIGHT (C) 2021 by the Society of Nuclear Medicine and Molecular Imaging. DOI: 10.2967/jnumed.121.262737 international, prospective, open label, multicenter, randomized phase 3 study of ${ }^{177}$ Lu-PSMA-617 in the treatment of patients with progressive PSMA-positive metastatic castration-resistant prostate cancer (VISION trial) showed that ${ }^{177} \mathrm{Lu}$-PSMA-617 significantly increased overall survival and radiographic progression-free survival in these patients (6). PSMA-based theranostics, therefore, very soon will increasingly become a clinical standard in prostate cancer patients - as long as these tumors express PSMA.

But PSMA is by far not as prostate-specific as suggested by its name. It is a type II transmembrane zinc metallopeptidase with enzymatic activity that hydrolyzes poly- $\gamma$-glutamated folates to folate, which can be taken up by nearby tumor cells (7). The enzyme is also known as glutamate carboxypeptidase II, folate hydrolase 1, folypoly- $\gamma$-glutamate carboxypeptidase, and $N$-acetylated- $\alpha$-linked acidic dipeptidase I. PSMA is physiologically expressed in astrocytes and Schwann cells of the nervous system, prostate, proximal renal tubule of the kidney, salivary glands, and the duodenal brush border $(8)$ as can be seen and quantified on PET images of cancer patients $(9)$.

In malignant tumors, however, PSMA expression is not only documented for prostate cancer cells but also found in the tumorassociated neovasculature of almost all solid tumors though not in normal vasculature. Interestingly, PSMA expression often correlates with the aggressiveness of tumors, as has been shown for prostate cancer as well as other tumor entities, for example, sarcomas in which PSMA expression was higher in more malignant tumors (10).

A comprehensive review on PSMA PET imaging of nonprostatic diseases has been recently compiled by de Galiza Barbosa et al., in which a variety of different tumors, including almost all relevant carcinomas as well as brain and nerve-derived tumors, lymphomas, and soft- and bone tissue sarcomas, were shown to be PSMA-positive, usually related to endothelial expression in the associated neovasculature. The authors therefore concluded that "these unintentional findings have paved the way for the application of PSMA PET imaging as an additional diagnostic tool" (11).

These "unintentional" findings of PET-detectable PSMA expression in many different malignant tumors imply potential use not only for imaging but also for therapy: it may open up a universal theranostic approach of tumor treatment in many tumors, and, thus, should be followed in more detail for different tumor entities, especially in tumors with a high medical need for therapy improvement.

Among all the nonprostatic PSMA-expressing tumors, aggressive brain tumor glioblastoma multiforme (GBM) holds special mention as treatment results in this tumor type, despite extensive research, are still very poor with no curative options thus far. Initial results in 16 
patients with histopathologically documented GBM showed PSMA expression, albeit highly heterogeneous, on both tumor-associated vessels and in nonendothelial cells (12). Similarly, further case reports and case series have also demonstrated mild to intense PSMA expression on PET/CT in GBM patients (13-18).

At first instance, these results appear promising, however, using a PSMA-based theranostic concept in glioblastoma may face several challenges based on these first reports. First, PSMA as the binding target is primarily expressed on neovascular rather than tumor cells in GBM, thus irradiating tumor cells only in the immediate vicinity of the vascular cells, depending on the range of the respective radionuclide. Second, the often low and diffusely distributed tumor volume in GBM recurrences with their infiltrating tumor cell clusters in comparison to, for example, solid metastatic prostate cancer lesions, may further reduce the therapeutic index. In this setting, the $\beta$-emitting radionuclide ${ }^{177} \mathrm{Lu}$, with a maximum pathlength of approximately $2 \mathrm{~mm}$, may release significant amounts of radiation to peritumoral brain parenchyma and nerves rather than to the infiltrating tumor cells, which in turn results in higher treatment toxicity. Finally, low to moderate PSMA expression in GBM may not allow sufficient tumor radiation doses if PSMA endoradiotherapy is used as a stand-alone treatment.

However, these limitations may be overcome. We first should perform clinical studies on PSMA PET/CT in correlation to conventional imaging, contrast-enhanced MRI, and ${ }^{18} \mathrm{~F}$-fluoroethyltyrosine or ${ }^{11} \mathrm{C}$-methionine PET, as well as tumor panel analysis for characterizing GBM lesions in terms of PSMA expression and the respective genotype. Second, we should use PSMA therapy with ${ }^{177} \mathrm{Lu}$ in the case of sufficient PSMA tumor uptake. Thorough state-of-the-art dosimetry in these patients in combination with meticulous tumor response assessment will allow evaluation of the clinical value of this treatment option as a stand-alone therapy and help define sufficient tumor uptake. On the basis of our above-mentioned caveats, combination treatments with stereotactic radiation therapy, sequentially or alternatively, or tyrosine kinase inhibitors should be evaluated early on in these highly treatment-resistant tumors. Furthermore, more effective therapeutic radionuclides, for example, ${ }^{161} \mathrm{~Tb}$ or the $\alpha$-emitter ${ }^{225} \mathrm{Ac}$ (very short pathlength!), have to be tested in comparison to ${ }^{177} \mathrm{Lu}$ for assessing both treatment efficacy and toxicity, and thus, their therapeutic index. Experience from radiation therapy suggests that apart from inherent radiation sensitivity of GBM cells, several other factors such as immune cell infiltration, radiation dose, duration of radiation therapy, and the like play a role in determining the treatment outcome. Radiolabeled PSMA binding to the neovasculature of GBM is expected to deliver relatively moderate radiation doses for inducing double-strand breaks in tumor cell DNA but at the same time modulate the tumor microenvironment to enhance tumor immunity as well as induce apoptosis through metabolic pathways. The radiobiology of PSMA endoradiotherapy of GBM needs to be properly investigated in appropriate preclinical and mathematic models.

It is most evident but imperative to state that such studies in GBM patients, either as prospective studies or under compassionate-use programs, have to be performed in interdisciplinary settings and close collaboration with neurosurgeons and radiation oncologists. Considering the extremely poor outcome of GBM patients, in terms of both survival and quality of life, the potential of PSMA radionuclide therapy should be explored for the benefit of these patients. Let us pave the way for PSMA theranostics to GBM and further tumor entities!

\section{DISCLOSURE}

No potential conflict of interest relevant to this article was reported.

\section{REFERENCES}

1. Hofman MS, Lawrentschuk N, Francis RJ, et al. Prostate-specific membrane antigen PET-CT in patients with high-risk prostate cancer before curative-intent surgery or radiotherapy (proPSMA): a prospective, randomised, multicentre study. Lancet. 2020;395:1208-1216.

2. Hofman MS, Murphy DG, Williams SG, et al. A prospective randomized multicentre study of the impact of gallium-68 prostate-specific membrane antigen (PSMA) PET/ CT imaging for staging high-risk prostate cancer prior to curative-intent surgery or radiotherapy (proPSMA study): clinical trial protocol. BJU Int. 2018;122:783-793.

3. Radzina M, Tirane M, Roznere L, et al. Accuracy of ${ }^{68}$ Ga-PSMA-11 PET/CT and multiparametric MRI for the detection of local tumor and lymph node metastases in early biochemical recurrence of prostate cancer. Am J Nucl Med Mol Imaging. 2020;10:106-118.

4. S3-Leitlinie Prostatakarzinom (version 6) (Guideline Prostate Cancer). Program Oncology c/o Deutsche Krebsgesellschaft e.V website. https://www.leitlinien programm-onkologie.de/leitlinien/prostatakarzinom/. Updated March 2021. Accessed July 23, 2021.

5. Lantheus Receives U.S. FDA approval of PYLARIFY® (piflufolastat F 18) injection, the first and only commercially available PSMA PET imaging agent for prostate cancer. Business Wire website. https://www.businesswire.com/news/home/20210527005291/ en/Lantheus-Receives-U.S.-FDA-Approval-of-PYLARIFY\%C2\%AE-piflufolastat-F18-Injection-the-First-and-Only-Commercially-Available-PSMA-PET-Imaging-Agentfor-Prostate-Cancer. Published online May 27, 2021. Accessed August 4, 2021.

6. Morris MJ, De Bono JS, Chi KN, et al. Phase 3 study of lutetium-177-PSMA-617 in patients with metastatic castration-resistant prostate cancer (VISION). J Clin Oncol. 2021;39(suppl 15):LBA4.

7. O'Keefe DS, Bacich DJ, Huang SS, Heston WDW. A perspective on the evolving story of PSMA biology, PSMA-based imaging, and endoradiotherapeutic strategies. J Nucl Med. 2018;59:1007-1013.

8. Vornov JJ, Peters D, Nedelcovych M, Hollinger K, Rais R, Slusher BS. Looking for drugs in all the wrong places: use of GCPII inhibitors outside the brain. Neurochem Res. 2020;45:1256-1267.

9. Prasad V, Steffen IG, Diederichs G, Makowski MR, Wust P, Brenner W. Biodistribution of ${ }^{68} \mathrm{GaPSMA}-\mathrm{HBED}-\mathrm{CC}$ in patients with prostate cancer: characterization of uptake in normal organs and tumour lesions. Mol Imaging Biol. 2016;18:428-436.

10. Heitkötter B, Trautmann M, Grünewald I, et al. Expression of PSMA in tumor neovasculature of high grade sarcomas including synovial sarcoma, rhabdomyosarcoma, undifferentiated sarcoma and MPNST. Oncotarget. 2017;8:4268-4276.

11. de Galiza Barbosa F, Queiroz MA, Nunes RF, et al. Nonprostatic diseases on PSMA PET imaging: a spectrum of benign and malignant findings. Cancer Imaging. 2020; 20:23.

12. Holzgreve A, Biczok A, Ruf VC, et al. PSMA expression in glioblastoma as a basis for theranostic approaches: a retrospective, correlational panel study including immunohistochemistry, clinical parameters and PET imaging. Front Oncol. 2021;11: 646387.

13. Unterrainer M, Niyazi M, Ruf V, Bartenstein P, Albert NL. The endothelial prostatespecific membrane antigen is highly expressed in gliosarcoma and visualized by ${ }^{68}$ Ga-PSMA-11 PET: a theranostic outlook for brain tumor patients? Neuro-oncol. 2017;19:1698-1699.

14. Salas Fragomeni RA, Pienta KJ, Pomper MG, Gorin MA, Rowe SP. Uptake of prostate-specific membrane antigen-targeted ${ }^{18} \mathrm{~F}$-DCFPyL in cerebral radionecrosis: implications for diagnostic imaging of high-grade gliomas. Clin Nucl Med. 2018;43: e419-e421.

15. Matsuda M, Ishikawa E, Yamamoto T, et al. Potential use of prostate specific membrane antigen (PSMA) for detecting the tumor neovasculature of brain tumors by PET imaging with ${ }^{89}$ Zr-Df-IAB2M anti-PSMA minibody. J Neurooncol. 2018;138:581589.

16. Salas Fragomeni RA, Menke JR, Holdhoff M, et al. Prostate-specific membrane antigen-targeted imaging with 18FDCFPyL in high-grade gliomas. Clin Nucl Med. 2017;42:e433-e435.

17. Sasikumar A, Kashyap R, Joy A, et al. Utility of ${ }^{68}$ Ga-PSMA-11 PET/CT in imaging of glioma-a pilot study. Clin Nucl Med. 2018;43:e304-e309.

18. Marafi F, Sasikumar A, Fathallah W, Esmail A. ${ }^{18}$ F-PSMA 1007 brain PET/CT imaging in glioma recurrence. Clin Nucl Med. 2020;45:e61-e62. 